\title{
EXPRESIÓN FENOTÍPICA in situ DE CARACTERÍSTICAS AGRONÓMICAS Y MORFOLÓGICAS EN POBLACIONES DEL MAÍZ RAZA JALA
}

\section{In situ PHENOTYPIC EXPRESSION OF AGRONOMIC AND MORPHOLOGICAL TRAITS IN POPULATIONS OF THE JALA LANDRACE}

\author{
Luis A. Montes-Hernández ${ }^{1}$, J. Arahón Hernández-Guzmán ${ }^{1 \star}$, Higinio López-Sánchez ${ }^{1}$, \\ Amalio Santacruz-Varela ${ }^{2}$, Humberto Vaquera-Huerta ${ }^{2}$ y Roberto Valdivia-Bernal ${ }^{3}$
}

\begin{abstract}
${ }^{1}$ Campus Puebla, Colegio de Postgraduados. Km. 125.5 Carr. Federal México-Puebla. 72760, Santiago Momoxpan, San Pedro Cholula, Puebla, México. Tel. (222) 2850013. ${ }^{2}$ Campus Montecillo, Colegio de Postgraduados, Km 36.5 Carr. México-Texcoco. 56230, Montecillo, Texcoco, Edo. de México, México. ${ }^{3}$ Unidad Académica de Agricultura, Universidad Autónoma de Nayarit. Km 9 Carr. Tepic-Compostela. 63780, Xalisco, Nayarit, México.
\end{abstract}

*Autor para correspondencia (arahon@colpos.mx)

\section{RESUMEN}

La conservación in situ de la diversidad genética del maíz (Zea mays L.) la han realizado los agricultores tradicionales durante años, mediante la siembra de poblaciones nativas como es el caso de la raza Jala, cuya conservación está amenazada por el crecimiento urbano y factores naturales, y actualmente en ella han disminuido características distintivas como altura de planta y longitud de mazorca. El número de agricultores que cultivan in situ poblaciones de la raza Jala es pequeño y con frecuencia intercambian entre ellos semilla de esta raza, por lo que es pertinente evaluar, con base en características morfológicas y agronómicas, si existe o no variación fenotípica entre poblaciones actuales de la raza Jala, conservadas y evaluadas en Jala, Nayarit, México, su lugar de origen y distribución primaria. Para la caracterización morfológica in situ, en 2010 se evaluaron 18 poblaciones del maíz raza Jala y siete testigos, en diseño Látice $5 \times 5$ con dos repeticiones, en tres ambientes ubicados en el Valle de Jala. Con el promedio de variables a través de ambientes se realizó análisis de varianza combinado, prueba de comparación de medias de Tukey, análisis de componentes principales(ACP) y análisis de conglomerados (ADC). El ACP definió que las variables con mayor influencia para explicar la variación observada fueron longitud de mazorca, altura de planta y altura de mazorca. El ADC delimitó tres grupos de poblaciones, en los que hubo mayor similitud fenotípica entre la mayoría de las poblaciones actuales. Se concluye que en la raza de maíz Jala existe poca variación fenotípica entre poblaciones actuales conservadas in situ, lo que presupone una base genética reducida y común.

Palabras clave: Zea mays, caracterización in situ, raza Jala, variación fenotípica.

\section{SUMMARY}

In situ conservation of genetic diversity in maize (Zea mays $\mathrm{L}$.) has been made for years by traditional farmers, through planting native populations, as is the case of the Jala landrace, whose preservation is threatened by urban growth and natural factors. The distinctive characteristics of this landrace, such as plant height and ear length, have become dimibished. The number of farmers who grow in situ population of the Jala landrace is low, and seed of this landrace is often exchanged among them. For these reasons, it is relevant to assess whether or not there is phenotypic variation among current, conserved populations of the Jala landrace in Jala, Nayarit, México, its place of origin and primary distribution. In situ morphological characterization of 18 Jala landrace populations and seven controls were evaluated in 2010 using a $5 \times 5$ Lattice design with two replications, at three environments within the Jala Valley. With the averages of variables through environments, a combined analysis of variance, Tukey's mean comparison, principal component analysis (PCA) and cluster analysis (ADC) were performed. PCA defined that the most influential variables for explaining the observed variation were ear length, plant height and cob height. ACD defined three groups of populations, in which there was greater phenotypic similarity with most of the current populations. It is concluded that in the Jala maize landrace there is low phenotypic variation among current in situ conserved populations, with a presumably reduced and common genetic base.

Index words: Zea mays, in situ characterization, Jala landrace, phenotypic variation.

\section{INTRODUCCIÓN}

La diversidad genética de una especie representa la variación heredable dentro y entre sus poblaciones, y en las especies cultivadas ésta tiene trascendencia pues es sobre la cual operan los procesos de selección que aplican los agricultores y fitomejoradores. Por ello es necesario caracterizarla, a fin de plantear esquemas más eficientes para su aprovechamiento y conservación (Romero y Muñoz, 1996).

Los estudios de diversidad genética de maíz (Zea mays L.) en su mayoría se han realizado en el continente americano (Sánchez et al., 2000b) y en países como México (Sánchez et al., 2000a), en donde las poblaciones nativas de maíz se siembran de manera regional, se cultivan en forma tradicional, y contribuyen a la conservación y generación de diversidad genética in situ (Bommer, 1991), misma que puede caracterizarse utilizando, de manera independiente o complementaria, marcadores morfológicos (Wellhausen et al., 1951), citogenéticos e isoenzimáticos (Sánchez et al., 2000a), o de ADN (Reif et al., 2006; Vigouroux et al., 2008).

Dentro de las razas de maíz clasificadas por Wellhausen et al. (1951) se encuentra la raza Jala, cuya característica 
distintiva es la longitud de mazorca (Kempton, 1924; Aguilar et al., 2006; Rice, 2006). Esta raza tiene importancia en la alimentación, tradición y cultura regional, aunado al potencial que representa su longitud de mazorca para mejorar otros maíces de mayor adaptación. De acuerdo con Kempton (1924), en 1907 el Valle de Jala contaba con alrededor de 300 ha de maíz nativo, cuyas plantas superaban los $6 \mathrm{~m}$ de altura y producían mazorca con longitudes superiores a los $50 \mathrm{~cm}$. En la década de los cuarenta a este tipo de maíz se le denominó raza Jala (Wellhausen et al., 1951), conocido localmente como Maíz de Húmedo.

En la actualidad, la superficie agrícola del valle de Jala se ha reducido por cambios en el uso del suelo (crecimiento urbano, construcción de autopistas, centros educativos, etc.) y se han introducido otras razas, poblaciones y variedades de maíz, así como otras especies cultivadas, de manera que, como lo documentó Rice (2006), la raza Jala ocupa menos de $5 \%$ del área dedicada al cultivo del maíz, lo que se traduce en una superficie no mayor a 30 ha; se siembra por menos de $20 \%$ de los agricultores de la región; la altura actual de planta es inferior a $4 \mathrm{~m}$ y también ha ocurrido reducción considerable en la longitud de su mazorca, y entre agricultores existe un importante intercambio de semilla de esta raza.

El objetivo de esta investigación fue caracterizar in situ, con base en atributos morfológicos y agronómicos, la variación fenotípica entre poblaciones actuales del maíz raza Jala, colectadas en tres localidades del área: Jala, Jomulco y Coapan, pertenecientes al municipio de Jala, Nayarit, México. El tener una referencia del grado de variación fenotípica existente permitirá sugerir opciones para su mantenimiento o ampliación, en su caso.

\section{MATERIALES Y MÉTODOS}

\section{Área de estudio}

La investigación se desarrolló en el Municipio de Jala, Nayarit, México, localizado entre $21^{\circ} 21^{\prime}$ y $21^{\circ} 03^{\prime} \mathrm{N}$ y entre $104^{\circ} 14^{\prime}$ y $104^{\circ} 33^{\prime} \mathrm{E}$, a una altitud promedio de 1080 m. El clima es del tipo Acw1 (semicálido subhúmedo, con lluvias en verano, de humedad media), con precipitación promedio anual de $837 \mathrm{~mm}$ (García, 1988). Las unidades de suelos predominantes son Cambisol (32.6\%), Regosol (26.75 \%), Leptosol (19.26 \%), Feozem (19.09 \%), Luvisol (2.71\%) y Andosol (0.05\%) (INEGI, 2014).

\section{Material genético}

Se evaluaron 18 poblaciones de la raza Jala (CPNAY1 al CPNAY18), y se incluyeron como testigos la accesión 2244 del CIMMYT, clasificada como raza Jala (identificada en lo sucesivo como CIMMYT); una población utilizada a nivel local denominada "San Juaneño", que aparentemente corresponde a una generación avanzada de algún híbrido comercial, cuya mazorca muestra similitud fenotípica a la de la raza Tuxpeño Norteño (CPNAY19); así como los compuestos COMPACC (derivado de la raza Jala y proporcionado por el Dr. Aquiles Carballo Carballo, Profesor Investigador del Colegio de Postgraduados), COMPGP (derivado de la raza Jala para producción de grano blanco para pozole), así como COMPRJC1, COMPRJC2 y COMPRJC3.

Los últimos tres materiales se obtuvieron de la siguiente manera: entre 1999 y 2007 se colectó la variación que del maíz raza Jala existía en la región. En 2007, con las 22 accesiones obtenidas se formó un compuesto balanceado y se sometió a recombinación en lote aislado; de este lote se seleccionaron 300 familias de medios hermanos fenotípicamente correspondientes a la raza Jala, con las cuales se formó el compuesto balanceado denominado COMPRJC1. En 2008, el COMPRJC1 se estableció en lote aislado para su recombinación genética, de donde fueron seleccionadas 300 familias de medios hermanos con las cuales se formó el COMPRJC2. Este proceso se repitió en 2009 y así se obtuvo el COMPRJC3. Los tres ciclos de selección y recombinación tuvieron lugar en el Valle de Jala.

\section{Establecimiento de experimentos y variables evaluadas}

Para llevar a cabo la caracterización fenotípica in situ, en abril de 2010 se establecieron en suelo tipo Feozem y condiciones de humedad residual, tres experimentos en igual número de ambientes, con diferencias entre ellos atribuibles de manera principal al manejo y a su ubicación dentro del valle de Jala. Uno de los sitios se localizó al centro del valle, a una altitud de $1050 \mathrm{~m}$, junto a un arroyo de corriente intermitente (sólo en temporal); en este sitio, durante los 4 años previos al establecimiento del experimento se incorporaron al suelo los restos de cosecha de maíz y malezas.

Un segundo sitio se ubicó a una altitud de $1057 \mathrm{~m}$, a una distancia aproximada de $120 \mathrm{~m}$ de donde termina el valle e inicia la parte cerril que lo circunda; durante los últimos años en este sitio se ha sembrado maíz de manera continua, sin incorporación al suelo de residuos de cosecha. El tercer sitio, con altitud de $1066 \mathrm{~m}$, se localizó en las cercanías de la comunidad de Jomulco, que se caracteriza por presentar textura al tacto ligeramente más arenosa que los otros sitios descritos, y en él se ha sembrado principalmente maíz, con rotaciones eventuales de cacahuate o garbanzo.

Se evaluaron 25 materiales genéticos mediante un diseño experimental Látice $5 \times 5$, con dos repeticiones. La unidad experimental constó de dos surcos de $0.8 \times 8 \mathrm{~m}$. 
Se depositaron 40 semillas por surco y en junio, ya establecida la temporada de lluvias, se realizó un aclareo para dejar 20 plantas por surco y una densidad de población de 31,250 plantas por hectárea. En todos los casos se fertilizó con el tratamiento $140 \mathrm{~N}-40 \mathrm{P}-00 \mathrm{~K}$, con urea y superfosfato de calcio triple, como fuentes de nitrógeno y fósforo, respectivamente. Todo el fósforo y $1 / 3$ parte del nitrógeno se aplicaron en la primera escarda, al momento en que se establecieron las lluvias. El resto del nitrógeno se aplicó en la segunda escarda.

Para la caracterización, en cada unidad experimental se registró la floración masculina y femenina en días después de la siembra, cuando $50 \%$ de espigas en la parcela experimental presentó emisión de polen y tenía estigmas expuestos en el jilote de la mazorca superior, respectivamente. Se etiquetaron cinco plantas con competencia completa en las cuales se cuantificó la altura de planta y mazorca en $\mathrm{cm}$, longitud total de espiga $(\mathrm{cm})$, longitud de ramas laterales primarias $(\mathrm{cm})$, longitud entre rama lateral inferior y superior $(\mathrm{cm})$, longitud de rama central $(\mathrm{cm})$ y de pedúnculo (cm), así como número de ramas de la espiga, a los $15 \mathrm{~d}$ después de ocurrida la floración.

A la cosecha, en noviembre de 2010, se colectaron las mazorcas de las plantas etiquetadas previamente, a las que se midió longitud y diámetro en $\mathrm{cm}$, se contó el número de hileras y granos por hilera, se determinó el porcentaje de grano y olote, y se tomaron 10 granos de la parte central para registrar la longitud, ancho y grosor en mm (HerreraCabrera et al., 2000). Además se determinó porcentaje de humedad y el factor de desgrane, el cual resultó de dividir peso del grano de las cinco mazorcas entre el peso del grano más olote de las mismas mazorcas. Se registró el peso de campo por unidad experimental $(\mathrm{kg})$ y se estimó el rendimiento por hectárea a $14 \%$ de humedad, ajustado por el factor de desgrane.

Las variables asociadas a planta, espiga y mazorca se promediaron por unidad experimental a través de ambientes para proceder a los análisis subsecuentes. A partir de las variables originales se generaron nuevas variables, para valorar su contribución adicional a la interpretación de resultados.

\section{Análisis estadístico}

Cada variable se sometió al análisis de varianza combinado a través de sitios de evaluación, para determinar los efectos de la interacción genotipo $\mathrm{x}$ ambiente. A las variables que resultaron significativas se les realizó la prueba de comparación de medias de Tukey mediante el procedimiento GLM del programa Statistical Analysis System $\mathbb{}$ (SAS, 2004). Luego se aplicó un análisis de componentes principales a partir de la matriz de correlaciones entre los atributos, con el procedimiento PRINCOMP (SAS, 2004). También se efectuó un análisis de conglomerados con la matriz de distancias euclidianas con el método de agrupamiento de mínima varianza dentro de grupos de Ward y el procedimiento CLUSTER (SAS, 2004). Para mejorar la presentación de la gráfica se aplicó el coeficiente de determinación como escala de disimilitud entre conglomerados (Mohammadi y Prasanna, 2003).

\section{RESULTADOS Y DISCUSIÓN}

En los Cuadros 1 y 2 se muestra el análisis de varianza combinado de variables originales y transformadas, evaluadas en poblaciones nativas establecidas en el Valle de Jala, Nayarit, en 2010. De las 35 variables consideradas en este análisis, hubo diferencias significativas $(\mathrm{P} \leq 0.05)$ entre genotipos en $25.7 \%$ de los casos, para ambientes las hubo en $85.7 \%$ de las variables, y en $45.7 \%$ de ellas para la interacción genotipo $\mathrm{x}$ ambiente.

Las variables asociadas directamente con rendimiento como las de grano, olote y mazorca fueron las que mostraron significancia estadística para la interacción genotipo $\mathrm{x}$ ambiente, como se ilustra en la Cuadro 1. Lo anterior indica que estos rasgos se expresan de manera diferente en ambientes contrastantes, como lo reportaron Aguilar et al. (2006). Por tanto son menos apropiadas para propósitos de caracterización fenotípica, comparadas con las variables derivadas de características morfológicas y fenológicas cuya interacción con el ambiente resultó no significativa. La variable ancho de grano (Cuadros 1 y 2) contrasta con los hallazgos de Wellhausen et al. (1951) y Aguilar et al. (2006), ya que el ancho de grano es superior, tal vez porque esta variable es de las más importantes que usan los agricultores para mantener la identidad de un maíz (Louette et al., 1997; Louette y Smale, 2000).

El Cuadro 3 muestra el análisis de componentes principales aplicado a los promedios de las 25 variables seleccionadas en las poblaciones evaluadas. Este análisis mostró que los tres primeros componentes en conjunto explicaron $75.9 \%$ de la variación fenotípica observada, donde el primer componente (CP1) con un valor característico de 6.45 explicó $41.9 \%$ del total de la varianza; el segundo componente (CP2), cuyo valor característico fue 4.67, explicó 17.5 $\%$, y el tercer componente (CP3) explicó $16.5 \%$ del total de la varianza.

Las variables originales con mayor influencia en el CP1 fueron longitud de mazorca, altura de mazorca y altura de planta/altura de mazorca. En el CP2 las variables originales de mayor valor discriminatorio fueron peso de mazorca, diámetro de mazorca y granos por hilera; mientras que para el 
Cuadro 1. Cuadrados medios y coeficientes de variación del análisis de varianza combinado de 26 variables morfológicas evaluadas en siete testigos y 18 poblaciones nativas del maíz raza Jala.Jala, Nayarit, México, 2010.

\begin{tabular}{|c|c|c|c|c|c|}
\hline \multirow{2}{*}{ Variable } & \multicolumn{5}{|c|}{ Cuadrados medios } \\
\hline & Gen & $\mathrm{Amb}$ & Gen $x$ Amb & Error & CV (\%) \\
\hline Altura de planta & $1484 \mathrm{~ns}$ & $49,516^{\star *}$ & $1256 \mathrm{~ns}$ & 1395 & 11 \\
\hline Altura de mazorca & $1337^{\star}$ & $42,144^{\star \star}$ & $686 \mathrm{~ns}$ & 679 & 12 \\
\hline Días a floración masculina & $28^{* *}$ & $408^{* *}$ & $7 \mathrm{~ns}$ & 10 & 3 \\
\hline Días a floración femenina & $21^{* *}$ & $466^{* *}$ & $1 \mathrm{~ns}$ & 3 & 2 \\
\hline Asincronía floral & $12^{*}$ & $19 \mathrm{~ns}$ & $7 \mathrm{~ns}$ & 7 & 70 \\
\hline Peso de olote & $12,298 \mathrm{~ns}$ & $9546 \mathrm{~ns}$ & $7368^{*}$ & 4025 & 26 \\
\hline Peso de mazorca & $245,972 \mathrm{~ns}$ & $266,666 \mathrm{~ns}$ & $197,902 * *$ & 100,505 & 27 \\
\hline Rendimiento & $499,423 \mathrm{~ns}$ & $16,831,191^{\star *}$ & $428,435^{* *}$ & 75,992 & 15 \\
\hline Factor de desgrane & $0.003 \mathrm{~ns}$ & $0.1^{* *}$ & $0.002^{* *}$ & 0 & 4 \\
\hline Número de espigas & $25 \mathrm{~ns}$ & $142^{\star *}$ & $19^{* *}$ & 8 & 13 \\
\hline Longitud de pedúnculo & $7 \mathrm{~ns}$ & $156^{* *}$ & $8 \mathrm{~ns}$ & 8 & 12 \\
\hline $\begin{array}{l}\text { Longitud entre rama lateral } \\
\text { inferior y superior }\end{array}$ & $14 \mathrm{~ns}$ & $496^{* *}$ & $14 \mathrm{~ns}$ & 10 & 7 \\
\hline Longitud de rama central & $7 \mathrm{~ns}$ & $54^{* *}$ & $9 \mathrm{~ns}$ & 9 & 9 \\
\hline Longitud de espiga & $44 \mathrm{~ns}$ & $855^{\star *}$ & $46 \mathrm{~ns}$ & 36 & 6 \\
\hline Longitud de ramas laterales & $7 \mathrm{~ns}$ & $13 \mathrm{~ns}$ & $11 \mathrm{~ns}$ & 8 & 10 \\
\hline Diámetro de mazorca & $18 \mathrm{~ns}$ & $42^{\star *}$ & $27^{\star *}$ & 5 & 4 \\
\hline Número de hileras & $1 \mathrm{~ns}$ & $1^{*}$ & $1^{* *}$ & 0.4 & 5 \\
\hline Granos por hileras & $45 \mathrm{~ns}$ & $163^{* *}$ & $40^{*}$ & 24 & 11 \\
\hline Longitud de mazorca & $11^{*}$ & $54^{* *}$ & $5^{*}$ & 3 & 8 \\
\hline Longitud de olote & $13^{*}$ & $88^{\star *}$ & $6^{*}$ & 3 & 8 \\
\hline Diámetro de olote & $11 \mathrm{~ns}$ & $5 \mathrm{~ns}$ & $7^{* *}$ & 3 & 5 \\
\hline Ancho de grano & 1 * & $3^{* *}$ & $0.4 \mathrm{~ns}$ & 0.3 & 4 \\
\hline Longitud de grano & $1 \mathrm{~ns}$ & $2^{\star *}$ & $1^{* *}$ & 0.5 & 5 \\
\hline Grosor de grano & $0.2 \mathrm{~ns}$ & $2^{* *}$ & $0.1 \mathrm{~ns}$ & 0.1 & 7 \\
\hline Peso de 100 granos & $61 \mathrm{~ns}$ & $1214^{\star *}$ & $47^{*}$ & 29 & 12 \\
\hline Volumen de grano & 10,570 * & $130,792^{\star *}$ & $5463 *$ & 3088 & 8 \\
\hline
\end{tabular}

${ }^{*} \mathrm{P} \leq 0.05 ;{ }^{* *} \mathrm{P} \leq 0.01 ; \mathrm{ns}=$ no significativa; Gen = genotipo; $\mathrm{Amb}=$ ambiente; Gen $\mathrm{x}$ Amb = genotipo $\mathrm{x}$ ambiente; $\mathrm{CV}=$ coeficiente de variación.

CP3 las variables originales de mayor importancia fueron longitud de ramas laterales, altura de planta más longitud de espiga/longitud de espiga, longitud de pedúnculo/longitud de espiga y longitud de la rama central/longitud de espiga. Estos resultados indican que los rasgos de espiga, grano y mazorca son importantes para describir el nivel de variación fenotípica existente entre las poblaciones evaluadas.

En el Cuadro 4 se muestran los promedios de los tres grupos obtenidos en el dendrograma generado por el análisis de conglomerados. El Grupo I presentó los promedios más bajos excepto en dos índices, mientras que los Grupos II y
III presentan diferencias en las características de altura de planta, altura de mazorca, longitud de mazorca y granos por hilera. Lo anterior permite aseverar que estos rasgos constituyen el criterio morfológico más importante para explicar la diversidad fenotípica existente entre los materiales genéticos evaluados. Para el caso de los componentes principales (Cuadro 3) y de los promedios de los nueve rasgos de los tres grupos obtenidos (Cuadro 4), se puede observar que las variables de mayor magnitud se asocian con características de espiga, grano y mazorca, las cuales se utilizaron para separar los grupos. Una muestra de la variacion fenotípica encontrada en esta caracteristicas se ilustra en la Figura 1. 


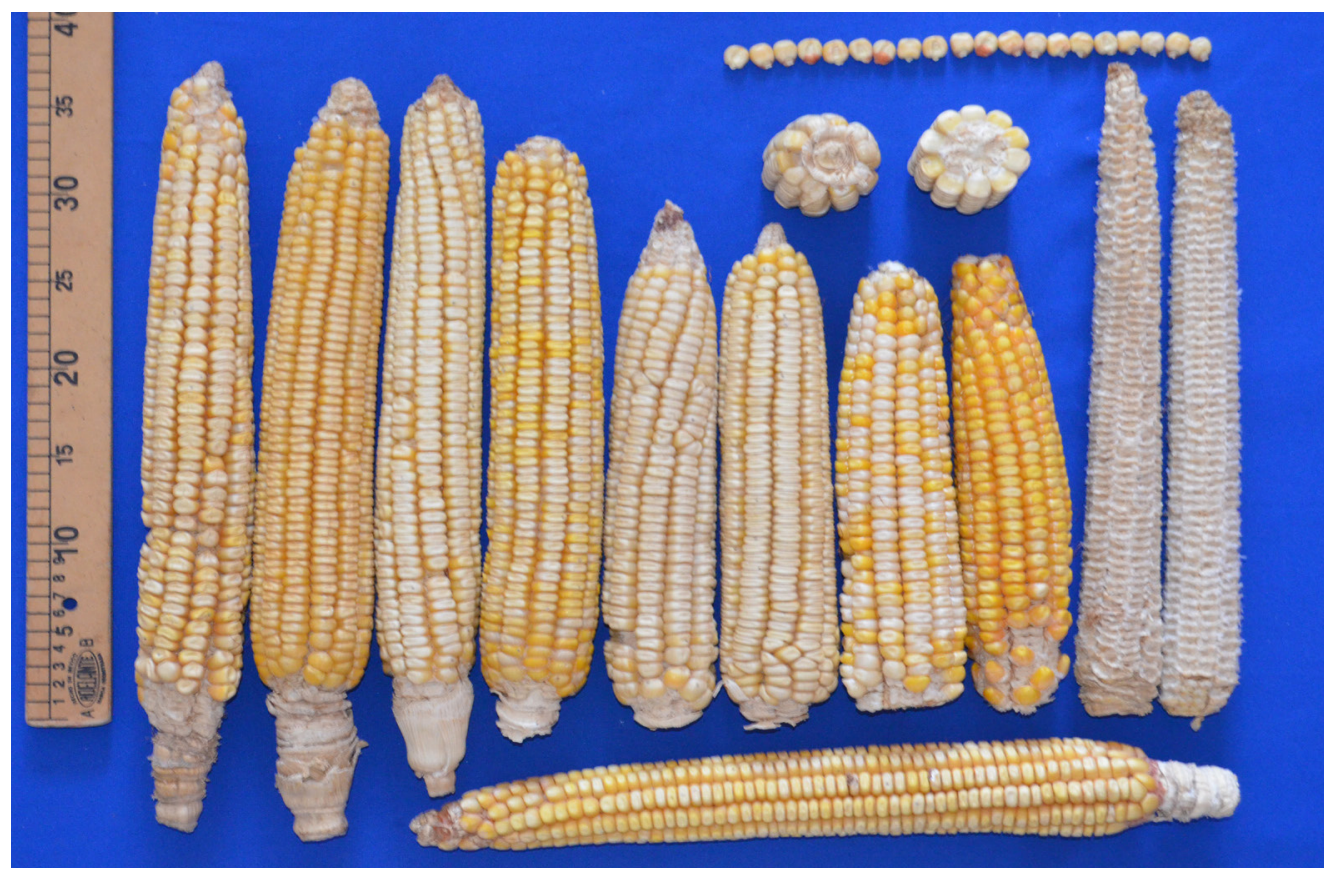

Figura 1. Muestra de la variación registrada en características de mazorca, grano y olote, entre las poblaciones evaluadas de la raza Jala.

Con la aplicación del análisis de conglomerados se obtuvo la Figura 2, que muestra la variación fenotípica entre los 25 materiales evaluados. Este análisis detectó los Grupos I, II y III que se formaron con dos, 10 y 13 genotipos, respectivamente, con base en las 25 variables que más contribuyeron a la variabilidad. El Grupo I, formado por la población San Juaneño (CPNAY19) y la accesión 2244 del CIMMYT, se caracterizó por su precocidad ( 80 y 83.5 d promedio a floración masculina y femenina, respectivamente), porte bajo de planta y posición baja de la mazorca.

La completa separación de ambos materiales del resto se explica en parte porque el genotipo CPNAY19, que se cultiva localmente por algunos agricultores, aparentemente es una generación avanzada de un híbrido, con características de mazorca similares a la raza Tuxpeño Norteño, más precoz, de menor porte y más rendidor que el maíz raza Jala; la accesión CIMMYT, por su parte, al mantenerse y regenerarse en ambientes que no corresponden a aquellos en donde prospera la raza Jala, probablemente sufrió cambios en su estructura genética, suficientes como para alejarse fenotípicamente de los atributos que caracterizan a las poblaciones de la raza Jala, mantenidas in situ.

Los materiales del Grupo II presentaron el ciclo más largo $(85$ y $88 \mathrm{~d}$ promedio a floración masculina y femenina, respectivamente) comparado con los otros dos grupos. El Grupo III se caracterizó por tener la mayor altura de planta y mazorca, así como peso de mazorca, diámetro de mazor- ca y longitud de mazorca. Las estructuras de espiga, grano y mazorca se constituyeron en los criterios morfológicos más importantes para la agrupación de las poblaciones, lo que coincidió parcialmente con las localidades donde éstas fueron colectadas, y esto sugiere adaptación de las mismas a esas condiciones particulares en donde la selección realizada por los agricultores a través de los años, además de que es fundamental, explica la disponibilidad de poblaciones nativas adaptadas a ambientes específicos (Romero y Muñoz 1996; Herrera-Cabrera et al., 2000; Ángeles-Gaspar et al., 2010).

De la Figura 2 destaca también el Grupo III que concentró $66.7 \%$ de las poblaciones actuales de la raza Jala, mientras que el resto de poblaciones se ubicaron en el Grupo II. La poca divergencia fenotípica entre poblaciones actuales conservadas in situ, presupone una base genética reducida y común, que en parte podría explicarse por el intercambio frecuente de semilla entre productores que conservan y cultivan a la raza Jala en su lugar de origen y principal distribución. El intercambio de semilla es un aspecto que se está perdiendo (Pressoir y Berthaud, 2004a, b; Perales et al., 2005), y que de acuerdo con Louette y Smale (2000) contribuye a generar y mantener la diversidad genética del maíz. Sin embargo, para el caso de la raza Jala, dicho intercambio se da entre un número muy reducido de agricultores, y en ocasiones es sólo la semilla de los agricultores que mejor mantienen el maíz raza Jala la que es requerida por otros agricultores. 
Cuadro 2. Cuadrados medios y coeficientes de variación del análisis de varianza combinado de nueve variables morfológicas evaluadas en siete testigos y 18 poblaciones nativas del maíz raza Jala. Jala, Nayarit, México, 2010.

\begin{tabular}{|c|c|c|c|c|c|}
\hline \multirow{2}{*}{ Variable } & \multicolumn{5}{|c|}{ Cuadrados medios } \\
\hline & Gen & $\mathrm{Amb}$ & Gen $x$ Amb & Error & $\mathrm{CV}(\%)$ \\
\hline Altura de planta/altura de mazorca & $0.04 \mathrm{~ns}$ & $0.9^{\star *}$ & $0.03 \mathrm{~ns}$ & 0.03 & 11 \\
\hline $\begin{array}{l}\text { Altura de planta + longitud de espiga/ } \\
\text { longitud de espiga }\end{array}$ & $0.24 \mathrm{~ns}$ & $10^{* *}$ & $0.2 \mathrm{~ns}$ & 0.22 & 10 \\
\hline $\begin{array}{l}\text { Longitud de pedúnculo/longitud de } \\
\text { espiga }\end{array}$ & $0 \mathrm{~ns}$ & $0.01 * *$ & $0 \mathrm{~ns}$ & 0 & 12 \\
\hline $\begin{array}{l}\text { Longitud de ramas laterales/longitud } \\
\text { de espiga }\end{array}$ & $0 \mathrm{~ns}$ & $0.003^{* *}$ & $0 \mathrm{~ns}$ & 0 & 8 \\
\hline $\begin{array}{l}\text { Longitud de rama central/longitud } \\
\text { de espiga }\end{array}$ & $0.01 \mathrm{~ns}$ & $0.01^{\star *}$ & $0 \mathrm{~ns}$ & 0 & 6 \\
\hline $\begin{array}{l}\text { Longitud de mazorca/diámetro de } \\
\text { mazorca }\end{array}$ & $0.11 \mathrm{~ns}$ & $0.8^{* *}$ & $0.1 \mathrm{~ns}$ & 0.04 & 9 \\
\hline Ancho de grano/longitud de grano & $0.004 \mathrm{~ns}$ & $0.01^{\star}$ & $0.01 * *$ & 0.002 & 6 \\
\hline Grosor de grano/longitud de grano & $0.002 \mathrm{~ns}$ & $0.01^{* *}$ & $0.002^{\star}$ & 0.001 & 11 \\
\hline Grosor de grano/ancho de grano & $0.001^{*}$ & $0.01 *$ & $0.001 \mathrm{~ns}$ & 0.001 & 8 \\
\hline
\end{tabular}

${ }^{*} \mathrm{P} \leq 0.05 ;{ }^{* *} \mathrm{P} \leq 0.01 ; \mathrm{ns}=$ no significativa; Gen = genotipo; $\mathrm{Amb}=$ ambiente; Gen $\mathrm{x} \mathrm{Amb}=$ genotipo $\mathrm{x}$ ambiente; $\mathrm{CV}=$ coeficiente de variación.

Por ello la contribución a la diversidad genética a través del intercambio de semilla pudiera resultar poco significante, debido a que en algún momento la semilla que se intercambia puede tener una base genética común. La alta similitud entre poblaciones actuales del maíz raza Jala puede explicarse también por el hecho de que la mayoría de los terrenos agrícolas destinados a su cultivo se localizan a distancias relativamente cercanas entre ellos, lo que impide un aislamiento apropiado, de modo que podría haber cruzamiento y recombinación entre poblaciones de maíz genéticamente cercanas. La poca divergencia fenotípica pudiera eventualmente poner en riesgo las posibilidades de adaptación y supervivencia del maíz raza Jala ante los cambios ambientales, cada vez más notorios en el Valle de Jala $\mathrm{y}$ áreas contiguas.

Los resultados sugieren reducida diversidad genética de la raza Jala, así como de sus características distintivas como son altura de planta y longitud de mazorca, según reportó Rice (2006). Las poblaciones correspondientes a la raza Jala se congregaron sólo en dos grupos, lo que evidencia la reducida variación fenotípica; en contraste, Aguilar et al. (2006) encontraron seis grupos en las localidades de Jala, lo que evidencia una mayor variación fenotípica. Puede afirmarse que en la raza Jala hay erosión genética, en la cual se involucra al ambiente debido a que éste tiene un papel fundamental en la estructura de la diversidad.

La formación de tres grupos y las diferencias en las distancias obtenidas con el método de Ward (Figura 2) permi- te suponer que hay una asociación entre el ambiente dado por sitio de evaluación y la diversidad fenotípica existente que fue menor. Las distancias relativamente pequeñas entre los grupos de las diferentes poblaciones sugieren una base genética común, posiblemente porque este maíz ha evolucionado principalmente en el Valle de Jala, con procesos similares de selección por parte de agricultores para características fisiológicas, de planta y de mazorca (Soleri y Cleveland, 2001; Pressoir y Berthaud, 2004b), así como para su adaptación natural a micronichos y gradientes altitudinales (Mercer et al., 2008; Ruiz-Corral et al., 2008).

Con el uso de microsatélites, Rice (2006) identificó alta similitud genética en las poblaciones actuales de la raza Jala que mantienen los agricultores de las comunidades de Jala, Jomulco y Coapan. Pressoir y Berthaud (2004a y 2004b) mencionan que en los sistemas tradicionales de Valles Centrales de Oaxaca, las características morfológicas como tamaño de mazorca, color de grano y periodo de floración, varían de acuerdo con el manejo que le da cada agricultor, pero con el uso de marcadores genéticos a nivel de genoma muestran similitud entre las poblaciones de maíz de una misma localidad.

Para estudios relevantes de diversidad en la razas de maíz, tanto a nivel inter-como intra-racial se han utilizado rasgos morfológicos como los reportados por Wellhausen et al. (1951), Herrera-Cabrera et al. (2000), López-Romero et al. (2005), Hortelano et al. (2008), Ángeles-Gaspar et al. (2010), entre otros. Este tipo de caracterización permite 
Cuadro 3. Vectores característicos, valor característico y proporción de varianza para los tres primeros componentes principales de 25 variables registradas de 18 poblaciones del maíz raza Jala y siete testigos evaluados en el municipio de Jala, Nayarit, México, en 2010.

\begin{tabular}{|c|c|c|c|}
\hline \multirow[b]{2}{*}{ Variable } & \multicolumn{3}{|c|}{ Vectores característicos } \\
\hline & $\begin{array}{l}\mathrm{CP} 1(6.45)^{\dagger} \\
{[41.9 \%]^{\dagger \dagger}}\end{array}$ & $\begin{array}{c}\mathrm{CP} 2(4.67)^{\dagger} \\
{[17.5 \%]^{\dagger \dagger}}\end{array}$ & $\begin{array}{c}\text { CP3 }(2.97)^{\dagger} \\
{[16.5 \%]^{\dagger \dagger}}\end{array}$ \\
\hline Longitud de espiga & 0.1867 & -0.1306 & 0.2765 \\
\hline Longitud de ramas laterales & 0.1465 & -0.2440 & $0.3268^{*}$ \\
\hline Peso de mazorca & 0.0389 & 0.2840 & 0.2750 \\
\hline Diámetro de mazorca & 0.1757 & 0.2965 & 0.1669 \\
\hline Número de hileras & -0.0384 & 0.1365 & -0.0013 \\
\hline Granos por hilera & 0.1350 & $0.3110^{*}$ & 0.0990 \\
\hline Longitud de mazorca & $0.3357^{\star}$ & 0.1284 & 0.0121 \\
\hline Peso de 100 granos & 0.2142 & 0.0702 & 0.2135 \\
\hline Altura de planta & $0.3070^{*}$ & 0.2511 & -0.2511 \\
\hline Altura de mazorca & $0.3234^{*}$ & 0.1208 & -0.1164 \\
\hline Días a floración masculina & 0.2467 & -0.2726 & -0.1672 \\
\hline Días a floración femenina & 0.2155 & -0.1704 & -0.0533 \\
\hline Asincronía floral & -0.1296 & 0.2165 & 0.1880 \\
\hline Factor de desgrane & -0.1140 & 0.1194 & -0.0462 \\
\hline Altura de planta/altura de mazorca & 0.2311 & 0.0334 & -0.0719 \\
\hline Longitud de mazorca/diámetro de mazorca & -0.2694 & 0.0513 & 0.0963 \\
\hline Altura de planta + longitud de espiga/longitud de espiga & 0.1162 & 0.2678 & $-0.3351^{\star}$ \\
\hline Longitud de pedúnculo/longitud de espiga & -0.1235 & 0.1760 & $0.3612^{*}$ \\
\hline Longitud de ramas laterales/longitud de espiga & 0.0201 & -0.1552 & 0.2245 \\
\hline Longitud de rama central/longitud de espiga & -0.0330 & -0.0222 & $0.3736^{*}$ \\
\hline Ancho de grano/longitud de grano & 0.1439 & -0.1620 & -0.0587 \\
\hline Grosor de grano/longitud de grano & 0.1805 & $-0.3190^{\star}$ & -0.1641 \\
\hline Grosor de grano/ancho de grano & 0.1218 & -0.2733 & -0.1578 \\
\hline Volumen de grano & 0.2442 & 0.1591 & 0.0991 \\
\hline
\end{tabular}

${ }^{\dagger}$ Valor característico del componente; ${ }^{\dagger}$ Porcentaje de varianza explicado por cada componente.

Cuadro 4. Promedios de nueve características de los tres grupos obtenidos en el dendrograma de 18 poblaciones de maíz raza Jala, y siete testigos en el valle del municipio Jala, Nayarit.

\begin{tabular}{cccccccccc}
\hline Grupo & ALPL & ALMZ & LMZ & GPH & GGLG & LRL & ALPLE & LPEDLE & LRCLE \\
\hline 1 & 298 & 165 & 18 & 39 & 0.35 & 27 & 4 & 0.24 & 0.33 \\
2 & 327 & 206 & 21 & 40 & 0.37 & 28 & 4 & 0.22 & 0.32 \\
3 & 339 & 209 & 22 & 44 & 0.35 & 27 & 4 & 0.23 & 0.32 \\
\hline
\end{tabular}

$\mathrm{ALPL}=$ altura de planta $(\mathrm{cm}) ; \mathrm{ALMZ}=$ altura de mazorca $(\mathrm{cm}) ; \mathrm{LMZ}=$ longitud de mazorca $(\mathrm{cm}) ; \mathrm{GPH}=$ granos por hilera; GGLG = grosor de grano/longitud de grano; LRL = longitud de ramas laterales $(\mathrm{cm}) ; \mathrm{ALPLE}=$ altura de planta + longitud de espiga/longitud de espiga; LPEDLE = longitud de pedúnculo/longitud de espiga; LRCLE = longitud de rama central/longitud de espiga. 


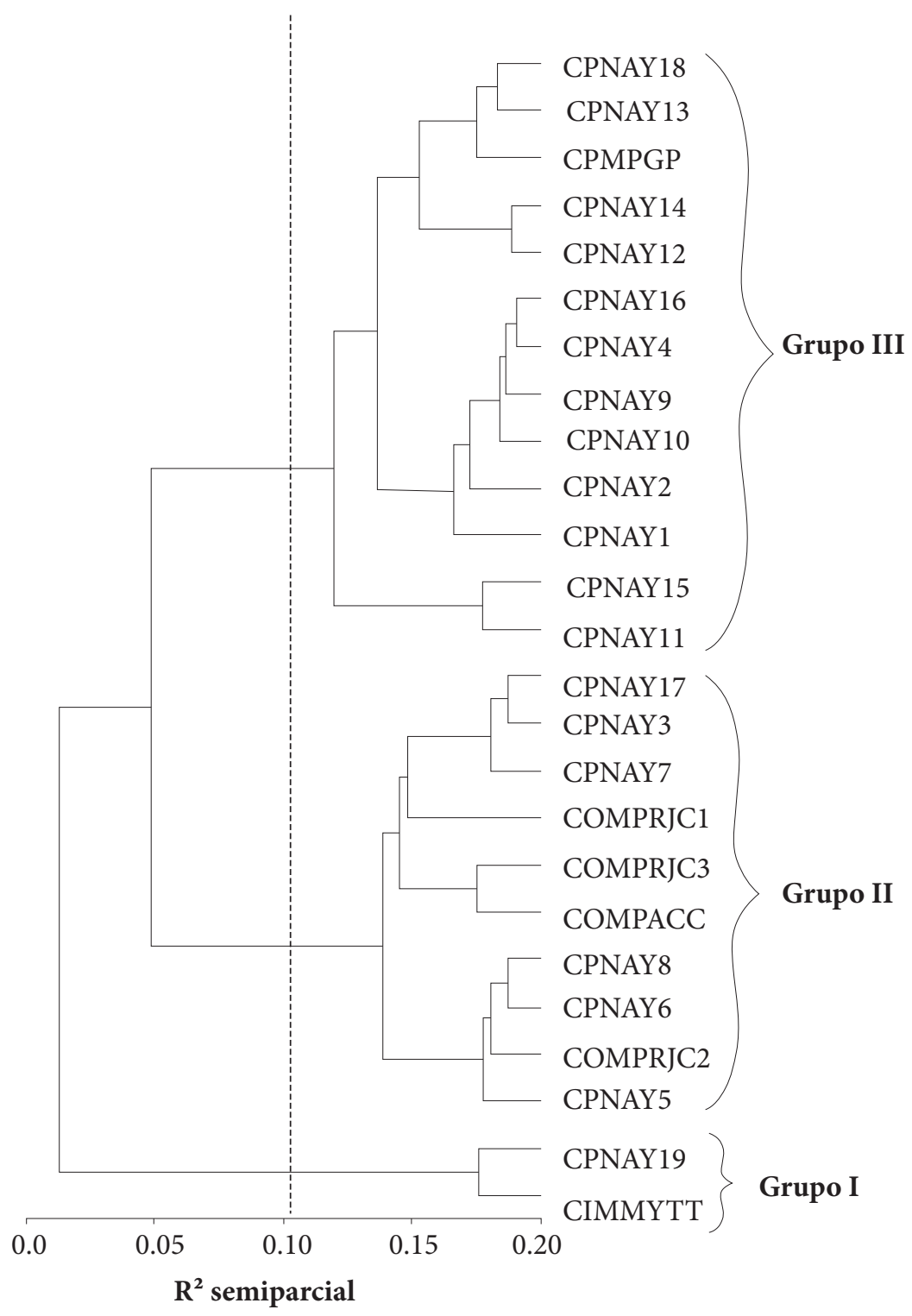

Figura 2. Dendrograma de 18 poblaciones de maíz y siete testigos, obtenido con el método de Ward.

conocer el proceso evolutivo de la diversidad genética a través de la variación de los órganos reproductivos y la determinación de sus patrones de variación, así como detectar la materia prima para los programas de mejoramiento genético y diseñar estrategias para la conservación.

El método empleado en esta investigación se considera útil para caracterizar la diversidad en las razas de maíz. Al respecto, se sugiere reunir las diferentes accesiones de la raza Jala conservadas en bancos de germoplasma, evaluarlas in situ junto con poblaciones actuales que los productores conservan in situ, seleccionar accesiones y poblaciones con características de planta y mazorca distintivas de la raza
Jala, utilizarlas para ampliar la base genética de esta raza, y llegar hasta la etapa de producción y distribución de semilla a productores interesados en su conservación. Esta metodología se puede complementar con el uso de marcadores moleculares y bioquímicos para tener una mayor precisión (Sánchez et al., 2000a; Vigoroux et al., 2008).

\section{CONCLUSIONES}

La evidencia experimental indica escasa divergencia fenotípica entre la mayoría de las poblaciones actuales de la raza Jala conservadas y caracterizadas in situ, lo que presupone una base genética reducida y común. 
Altura de planta, altura de mazorca y longitud de mazorca fueron los criterios morfológicos más importante para explicar la variación fenotípica existente entre los materiales genéticos evaluados; mientras que las características de grano y mazorca aunadas a las estructuras de espiga tuvieron la mayor contribución en su agrupación.

\section{AGRADECIMIENTOS}

Al Consejo Nacional de Ciencia y Tecnología (CONACYT), por la beca doctoral otorgada al primer autor. Al Servicio Nacional de Inspección y Certificación de Semillas (SNICS), por el financiamiento que a través del Sistema Nacional de Recursos Fitogenéticos para la Alimentación y la Agricultura (SINAREFI), ha venido otorgando para la conservación in situ de la raza Jala, lo cual facilitó el desarrollo de la presente investigación; y en este mismo sentido, al Colegio de Postgraduados, Campus Puebla. Al Ing. Israel Ruvalcaba Rivas, por el apoyo en la conducción de experimentos.

\section{BIBLIOGRAFÍA}

Aguilar C. J. A., A. Carballo, F. Castillo, A. Santacruz, A. Mejía, J. Crossa y G. Baca (2006) Diversidad fenotípica y variantes distintivas de la raza Jala de maíz. Agricultura Técnica en México 32:57-66.

Ángeles-Gaspar E., E. Ortiz, P. A. López y G. López (2010) Caracterización y rendimiento de poblaciones de maíz nativas de Molcaxac, Puebla. Revista Fitotecnia Mexicana 33:287-296.

Bommer D. F. R. (1991) The historical development of international collaboration in plant genetic resources. In: Searching for New Concepts for Collaborative Genetic Resources Management. Th. J. L. Van Hintun, L. Frese and P. M. Perrer (eds.). Papers of the EUCARPIA/IBPGR Symposium. International Board for Plant Genetic Resources. Roma, Italia. pp:3-12.

García E. (1988) Modificaciones al Sistema de Clasificación Climática de Köpen. $4^{\mathrm{a}}$ ed. México, D.F. 219 p.

Herrera-Cabrera B. E., F. Castillo, J. J. Sánchez, R. Ortega y M. Goodman (2000) Caracteres morfológicos para valorar la diversidad entre poblaciones de maíz en una región: caso la raza Chalqueño. Revista Fitotecnia Mexicana 23:335-354

Hortelano S. R. R., A. Gil, A. Santacruz, S. Miranda y L. Córdova (2008) Diversidad morfológica de maíces nativos en el Valle de Puebla. Agricultura Técnica en México 34:189-200.

INEGI, Instituto Nacional de Estadística, Geografía e Informática (2014) Compendio de Información Geográfica Municipal. INEGI. México, D.F. Disponible en: http://www.inegi.org.mx/ geo/contenidos/topografia/compendio.aspx (Junio 2014).

Kempton J. H. (1924) Jala maize: A giant variety from Mexico. Journal of Heredity 15:337-344.
López-Romero G., A. Santacruz-Varela, A. Muñoz-Orozco, F. CastilloGonzález, L. Córdoba-Téllez y H. Vaquera-Huerta (2005) Caracterización morfológica de poblaciones nativas de maíz del Istmo de Tehuantepec, México. Interciencia 30:284-290.

Louette D., A. Charrier and J. Bertaud (1997) In situ conservation of maize in Mexico: genetic diversity and maize seed management in a traditional community. Economic Botany 51:20-38.

Louette D. and M. Smale (2000) Farmers' seed selection practices and traditional maize varieties in Cuzalapa, México. Euphytica 113:25-41.

Mercer K., A. Martínez-Vásquez and R. Perales (2008) Asymmetrical local adaptation of maize landraces along an altitudinal gradient. Evolutionary Applications 1:489-500.

Mohammadi S. A. and B. M. Prasanna (2003) Analysis of genetic diversity in crop plants-salient statistical tools and considerations. Crop Science 43:1235-1248.

Perales H. R., B. F. Benz and S. B. Brush (2005) Maize diversity and ethno-linguistic diversity in Chiapas, Mexico. Proceedings of the National Academy of Sciences USA 102:949-954.

Pressoir G. and J. Berthaud (2004a) Patterns of population structure in maize landraces from the Central Valleys of Oaxaca in Mexico. Heredity 92:88-94.

Pressoir G. and J. Berthaud (2004b) Population structure and strong divergent selection shape phenotypic diversification in maize landraces. Heredity 92:95-101.

Reif J. C., L. Waburton, C. Xia, A. Hoisington, J. Crossa, S. Taba, J. Muminovic, M. Bohn, M. Frisch and E. Melchinger (2006) Grouping of accessions of Mexican races of maize revisited with SSR markers. Theoretical and Applied Genetics 113:177185.

Rice E. B. (2006) Conservation and change: a comparison of in-situ and ex-situ conservation of Jala maize germplasm. Crop Science 46:428-436.

Romero P. J. y A. Muñoz (1996) Patrón varietal y selección de variedades de maíz para los sistemas en la región de tierra caliente. Agrociencia 30:63-73.

Ruiz-Corral J. A., N. Duran-Puga, J. J. Sánchez-González, J. Ron-Parra, D. R. González-Eguiarte, B. Holland J. and G. Medina-García (2008) Climatic adaptation and ecological descriptors of 42 mexican maize races. Crop Science 48:1502-1512.

Sánchez G. J. J., M. Goodman and C. W. Stuber (2000a) Isozymatic and morphological diversity in the races of maize of Mexico. Economic Botany 54:43-59.

Sánchez G. J. J., M. Goodman and C. W. Stuber (2000b) Isozymatic diversity of the races of maize of the Americas. Maydica 45:185203.

Soleri D, and A. Cleveland (2001) Farmers' genetic perception regarding their crop populations: an example with maize in the Central Valleys of Oaxaca, Mexico. Economic Botany 55:106-128.

SAS (2004) The SAS ${ }^{\circledR}$ System for Windows ${ }^{\circledR}$ (Ver. 9.0). SAS Institute Inc. Cary, NC.

Vigouroux Y., J. C. Glaubitz, Y. Matsuoka, M. Goodman, J. Sánchez and J. Doebley (2008) Population structure and genetic diversity of New World maize races assessed by DNA microsatellites. American Journal of Botany 95:1240-1253.

Wellhausen E. J., L. M. Roberts, E. Hernández y C. Mangelsdorf (1951) Razas de Maíz de México. Su Origen, Características y Distribución. Folleto Técnico No. 5. Oficina de Estudios Especiales. Secretaría de Agricultura y Ganadería. México, D.F. 